\title{
MoviBed - Sleep Analysis Using Capacitive Sensors
}

\author{
Maxim Djakow ${ }^{1}$, Andreas Braun ${ }^{2}$, and Alexander Marinc ${ }^{2}$ \\ ${ }^{1}$ Hochschule Darmstadt, Darmstadt, Germany \\ maxim.djakowastud.h-da.de \\ ${ }^{2}$ Fraunhofer Institute for Computer Graphics Research IGD, Darmstadt, Germany \\ \{andreas.braun, alexander marinc\} @igd. fraunhofer. de
}

\begin{abstract}
Sleep disorders are a wide-spread phenomenon that can gravely affect personal health and well-being. An individual sleep analysis is a first step in identifying unusual sleeping patterns and providing suitable means for further therapy and preventing escalation of symptoms. Typically such an analysis is an intrusive method and requires the user to stay in a sleep laboratory. In this work we present a method for detecting sleep patterns based on invisibly installed capacitive proximity sensors integrated into the bed frame. These sensors work with weak electric fields and do not disturb sleep. Using the movements of the sleeping person we are able to provide a continuous analysis of different sleep phases. The method was tested in a prototypical setup over multiple nights.
\end{abstract}

Keywords: Capacitive proximity sensor, sleep analysis, smart furniture.

\section{Introduction}

Sleeping is the single most time-consuming activity of our lives - on average we spend approximately a third of our lives sleeping. It is peculiar that our knowledge regarding this very important activity remains limited. However, its importance becomes apparent as soon as sleep deprivation sets in. Work performance, well-being, concentration are negatively affected almost immediately, and may lead to severe long-term consequences if the situation is not remedied [1].

Sleep can be distinguished into different phases, their frequency and duration forming the basis for measuring the sleep quality of an individual person [2]. A popular method to distinguish those phases is based on recognizing the movement of a person throughout the night, a so called actigraphy [3]. The advent of small accelerometers based on microelectromechanical systems (MEMS) has made it possible to perform this type of screening at home, e.g. using wristbands or smartphones [4]. However, it still requires either a body-worn device or an external unit that is attached to the bed.

Capacitive proximity sensors allow detecting the presence of a human body by means of weak oscillating electric fields. They have been applied to various applications for body parameter sensing in fields such as human-computer interaction or smart furniture, creating different interaction devices, or furniture that is able to sense occupation and posture [5-7]. 
In this work we present MoviBed, an extension to one of our earlier prototypes [8]. This earlier prototype allowed detecting position and posture of one or two persons on a bed, based on static analysis of capacitive proximity sensors data. MoviBed allows the detection of sleep phases based on movement registered by an array of capacitive sensors that is attached to the bed frame. It uses dynamic data analysis to gather a measure of overall movement to detect sleep phases, as well as additional data regarding single movements. The system was installed in an extended prototype and evaluated for both detection of different movements and the analysis of different sleep phases.

\section{Related Works}

The most common method and "gold standard" for determining sleep phases is producing a polysomnogram in a sleep laboratory. These devices are monitoring a large number of body activities, the most common being electrical brain activity (EEG), electrical muscle activity (EMG), eye movement (EOG), hear rate (ECG), breathing rate, acoustic detection of snoring, body posture, leg movement and blood oxygen levels. The result is a graph as shown in Fig. 1. It allows a precise analysis of various sleep disorders and distinguishing the different sleep phases. However, the equipment is quite invasive and requires the sleeping person being attached to numerous measuring instruments.

The recent advent of mobile technology has spawned numerous smartphones equipped with MEMS systems and microphones that can be used to detect movement in the sleep $[4,9]$. The phone is usually placed on the mattress, e.g. below the pillow

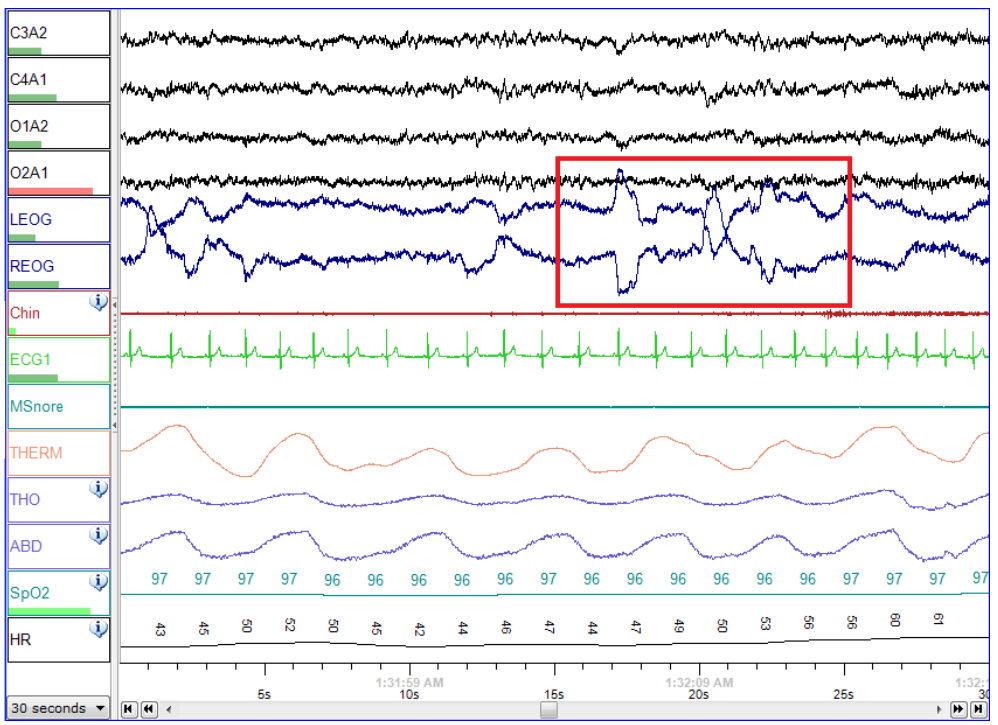

Fig. 1. Polysomnogram of a person in REM sleep (Source: http://en.wikipedia.org/wiki/ File:Sleep_Stage_REM.png) 


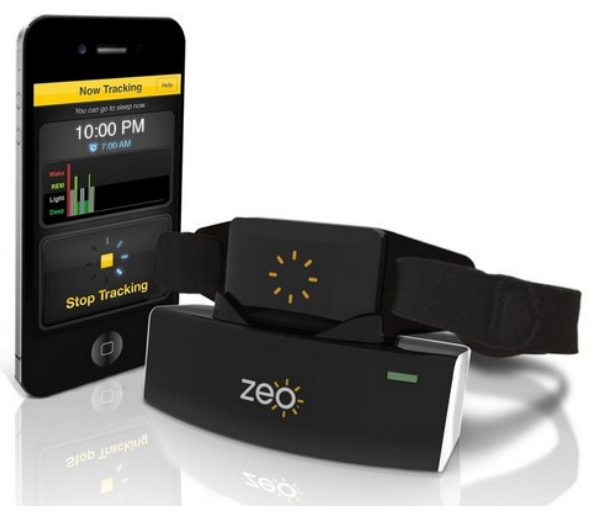

Fig. 2. Zeo sleep manager with electrode headband, base station and smartphone running the associated application

and tracks movement either by vibrations of the mattress or sounds the user generates when moving. A variety of personal health applications are available on all modern smartphone operating system, one example being WakeApp, providing an improved wake experience by adjusting alarm time according to the currently detected sleep phase [10].

A final category is made up of devices specifically designed for personal sleep analysis. Those provide a middle ground between intrusive laboratory environment and light-weight analysis by smartphones. One example is the Zeo Sleep Manager shown in Fig. 2. This device is comprised of an electrode headband that provides EEG measurements, a base station that is receiving and analyzing those signals and various applications for PC and smartphone that provide user interfaces for personal sleep analysis. Additionally there is a social networking component that allows comparing the individual sleep quality with other users of the system.

\section{$3 \quad$ Sleep Phase Recognition}

The most reliable way to track sleep phases is by using an electroencephalography (EEG); that is measuring the electrical activity of the brain by placing electrodes on the scalp. Various different types of neural oscillations can be distinguished - the most important for sleep phase detection are alpha waves, theta waves, delta waves and sleep spindles. The American Academy of Sleep Medicine (AASM) distinguishes three different phases of non-rapid eye movement sleep (NREM) and REM phase [11].

- Stage 1 - occurs mostly in the beginning of sleep. It has slow eye movement, alpha waves disappear and the theta wave appears.

- Stage 2 - dreaming is very rare and no eye movement occurs. The sleeper is quite easily awakened. EEG recordings have a tendency for characteristic "sleep spindles" 
- Stage 3 - was previously divided into stages 3 and 4. It is slow-wave sleep (SWS) or deep sleep. Stage 3 used to be the transition between stages 2 and 4 where delta waves began to occur, while delta waves are dominant in stage 4 .

- REM sleep - is a phase of sleep characterized by random and rapid movement of the eyes. It is considered the lightest phase of sleep and occurs all through the night but gets longer close to morning.

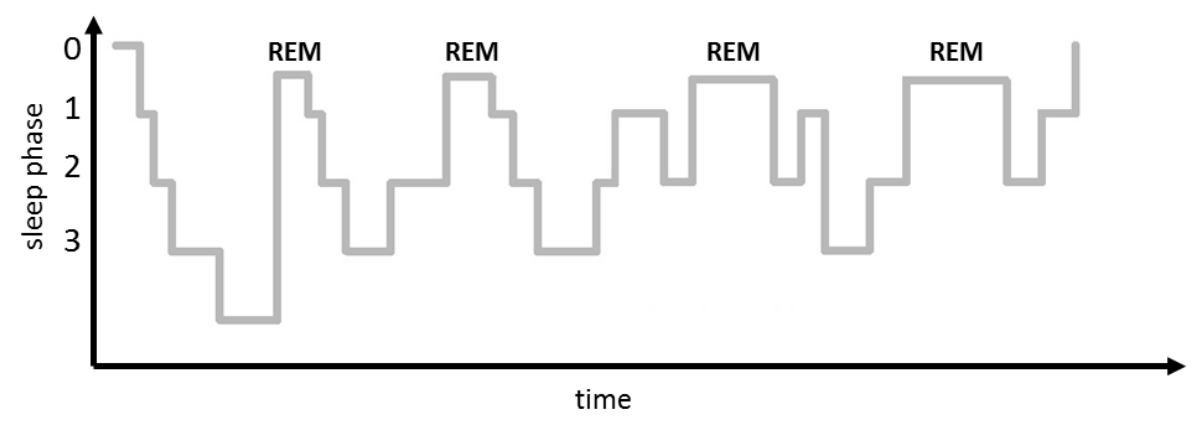

Fig. 3. Example of human sleep phases throughout the night

A typical distribution of sleep phases throughout the night is shown in Fig. 1. It can be easily scene that the sleep is distributed into different cycles, whereas the sleeping person is moving through the different sleep phases until having a REM phase and then going back to deep sleep. If the only available data is body movements it is becoming more difficult to reliably determine the sleep phase. Studies have shown that the magnitude of movement is typically associated to the following phases in decreasing order: wake, stage 1, REM, stage 2, stage 3 [12]. Another method is distinguishing between awake phase, active sleep and quite sleep and takes into account the order of those phases. This information allows to correlate the actual sleep phases with good certainty [13]. We have chosen this method for our system.

\section{Movement-Detection Using Capacitive Sensors}

Capacitive proximity sensors enable us to detect the presence of suitable object and their relative proximity to the electrode. Consequently a moving object will cause a change of sensor values. If we aggregate these data deviations from an array of sensors we get a reliable measure of objects moving above the electrodes. In the case of MoviBed we can assume that there is a limited number of persons moving on top of the sensors and thus it is possible to associate the sensor values to movement. In the following we will present a suitable method to achieve a reliable detection of the movements of a sleeping person. We are following a similar approach as Salmi and Leinonen [13].

At any given time $t$ a set of the latest values of all $n$ sensors can be stored as a tuple in the following form: 


$$
\overrightarrow{s_{t}}=\left(\begin{array}{c}
s_{1_{t}} \\
s_{2} \\
\vdots \\
s_{n_{t}}
\end{array}\right)
$$

As capacitive proximity sensors are particularly susceptible to external influences, such as temperature, humidity and other electric fields it is necessary to apply filtering on the sensor values. A suitable candidate is a median filter - a low-pass filter method that selects the median object of a sorted set of values, thus discarding outliers and strongly deviating values. This is particularly suited if transmission errors may occur.

If a person is moving on the bed the value of all sensors in detection distance of the moved body parts will change accordingly, the most relevant example in our case being a person moving in its sleep. We can generate a measure of movement intensity by comparing the values at time $t$ with those at time $t-1$ resulting in:

$$
\overrightarrow{d_{t}}=\left|\overrightarrow{s_{t}}-\overrightarrow{s_{t-1}}\right|=\left(\begin{array}{c}
\left|s_{1_{t}}-s_{1_{t-1}}\right| \\
\left|s_{2_{t}}-s_{2_{t-1}}\right| \\
\vdots \\
\left|s_{n_{t}}-s_{n_{t-1}}\right|
\end{array}\right)
$$

In subsequent calculations we will use $\overrightarrow{d_{t}}$ as combined measurement. For distinguishing between wake, active sleep and quiet sleep we are solely interest in the most intense movement. Thus we are testing for the largest value over a set of $m$ samples, generating the value $b_{t}$.

$$
b_{t}=\max \left(\overrightarrow{d_{t 1}}, \overrightarrow{d_{t 2}}, \ldots, \overrightarrow{d_{t m}}\right)
$$

The value $b_{t}$ is affected by changes in the speed of movement. Therefor as a final step we generate a centered average value of order $2 q-1$ :

$$
\bar{b}_{t}=\frac{1}{2 q-1} \sum_{i=-q}^{q} b_{t-i}
$$

The resulting value $\overline{b_{t}}$ allows us to quantify the intensity of movements over a given period. In order to extract an actual body movement from this value we have to quantify a threshold $s(t)$ that is determined by the average of $q$ previous values of $\overline{b_{t}}$ multiplied with a factor $f$ that has to be evaluated individually for each configuration of bed and sensors. This threshold $s(t)$ allows us to identify a movement $m$ at any time $t$. This behavior is denoted in the following equations:

$$
s(t)=\left(\frac{1}{q} \sum_{i=1}^{q+1} \overline{b_{t-l}}\right) * f, \quad m_{t}=\left\{\begin{array}{l}
1, \text { if } \overline{b_{t}}>s(t)>\overline{b_{t-1}} \\
0, \text { else }
\end{array}\right.
$$

As previously mentioned it is difficult to determine sleep phases solely by monitoring the movement. Instead following the example of Salmi and Leinonen and distinguish three phases - wake, active sleep and quiet sleep [13]. These are determined by dividing the sleep time into $a$ three-minute epochs $e_{i_{a}}$ and qualify these as active or quiet by counting the number of movements occurring in those intervals and 
comparing it to the average amount of movements in all epochs $\overline{e_{a}}$ determined by the following equations:

$$
e_{i_{a}}=\sum_{e_{i_{\text {Start }}}^{e_{\text {End }}}}^{e_{i}}, \quad \overline{e_{a}}=\frac{1}{n} \sum_{i=0}^{n} e_{i_{a}}
$$

In consequence we determine the status of any epoch with this final equation:

$$
e_{i_{a}}=\left\{\begin{aligned}
& \text { active, } \text { if } e_{i_{a}}>\overline{e_{a}} \\
& \text { quiet, } \text { if } e_{i_{a}} \leq \overline{e_{a}}
\end{aligned}\right.
$$

These active and quiet periods can be semi-autonomously interpreted by humans in order to determine the actual sleep phases. For example initial activity for 20 to 40 minutes followed by a quiet period can be attributed to a person falling asleep. Following quiet phases are a good indicator for deep sleep phases.

\section{MoviBed Prototype}

The Prototype uses existing hardware that has been created by Braun et al. [8] that uses the CapToolKit [14] to read from eight capacitive sensors and send their values to a PC using USB. Each of the sensors uses thin copper-foil as electrodes that are attached to the slatted frame of the bed, as shown in Fig. 4. On each bedside is one electrode placed below the head-area, two below the top and bottom torso-area and one below the feet. This way the whole body can interact with the sensors and most motions can be detected. The computer besides the bed runs a Java-program which reads the character-string encoded sensor-values via the RXTX library from the serial interface and processes the data in the way described in the previous chapter. To be read and analyzed later by this or other programs, all processed data of a recording can be saved in a comma-separated-file, together with its associated time and date, detected motions and epochs. For further investigation by a human gets the processed data in a simple GUI visualized.

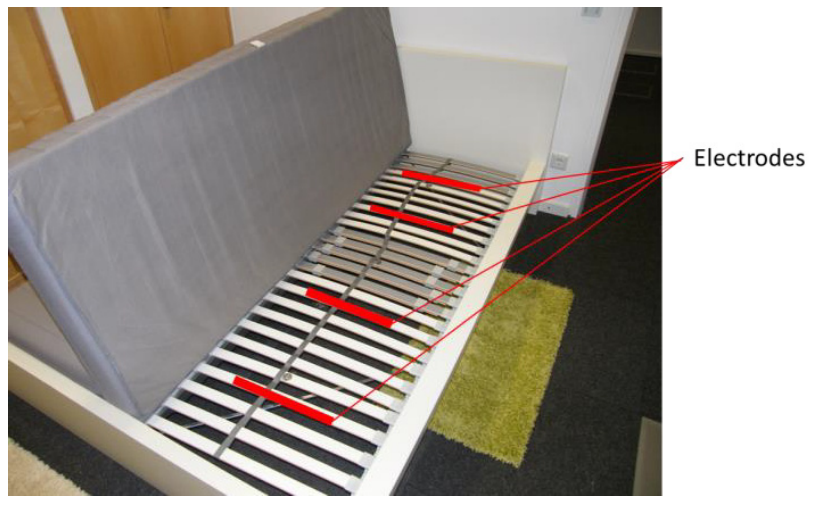

Fig. 4. MoviBed prototype [8] 


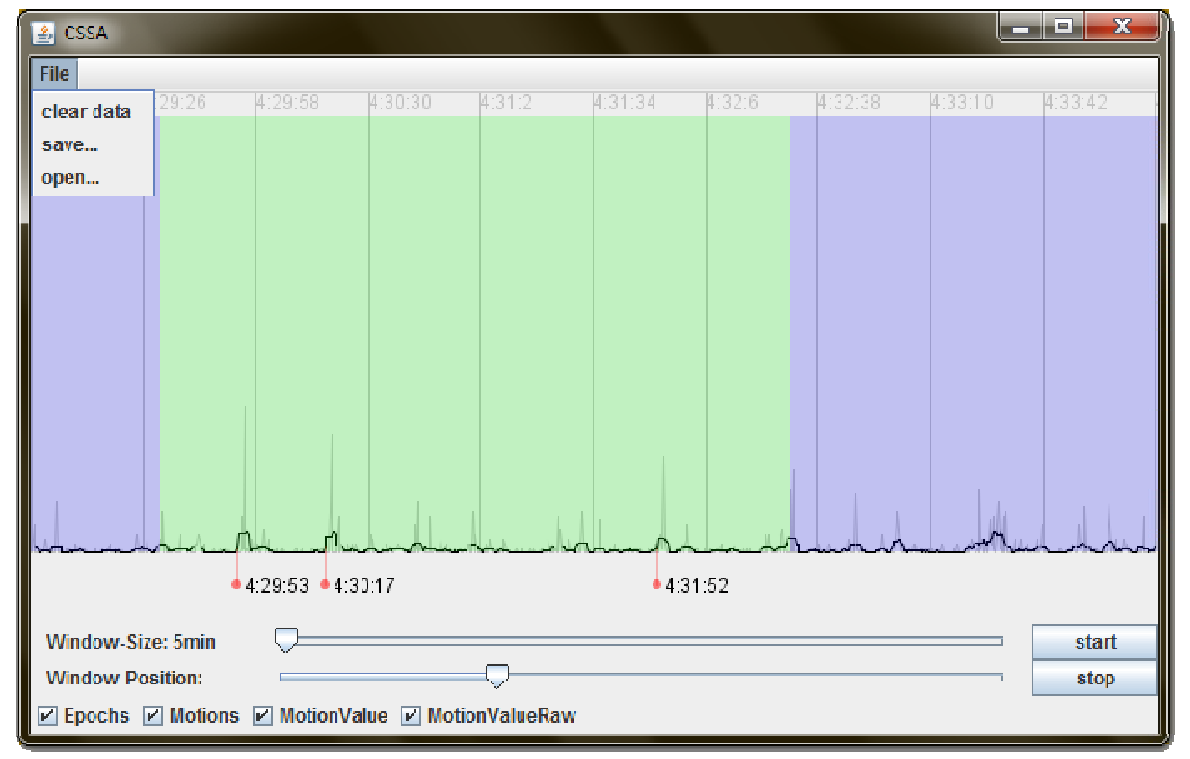

Fig. 5. Graphical user interface of the prototype

All movement-data are displayed in real-time in a linear graph, each detected motion is marked and labeled with its time and the epochs are visualized as coloring of the graphs background, in which active phases are shown in green and quiet phases in blue. This way the whole recording can be analyzed at one glance or in smaller timewindows for a more detailed analysis.

\section{Evaluation}

We have performed two different evaluations of MoviBed. The first part is a test of the detection precision regarding different movements. The test person was performing the following chain of events: (1) Lying down and standing up again, (2) moving the left and right arms away from the body horizontally, (3) moving the arms up and down, (4) moving the left and right leg away from the body horizontally, (5) lifting the left and right leg, (6) rotating the head, (7) rotating the whole body. The test person was asked to perform this sequence waiting 15 seconds between each event. Distinct values $b_{t}$ were recorded over time and plotted to get a measure for the detected movement intensity.

Fig. 2 gives an overview of some example sensor activities caused by (1) moving an arm and (2) moving the head alone. It can be observed that the head is causing a considerably smaller output compared to the arm. This can be attributed to the fact that the head is a smaller object and the movement does barely alter the shape of the body with respect to the sensor electrodes. Therefore we can group the movements into three different categories based on how distinct the sensor response is: 


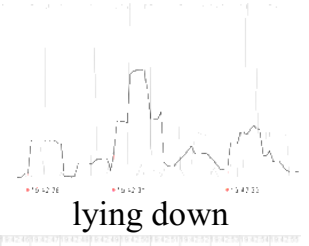

moving the left arm horizontally

moving the left arm up and down

moving the left leg horizontally

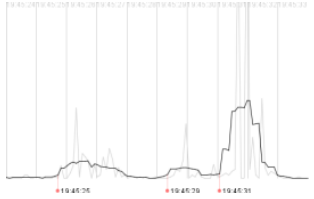

standing up

moving the right arm horizontally

moving the right arm up and down

moving the right leg horizontally

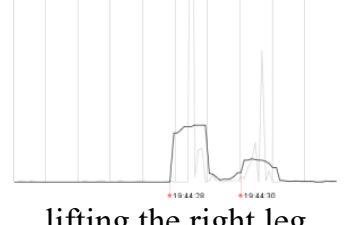

lifting the right leg

lifting the left leg

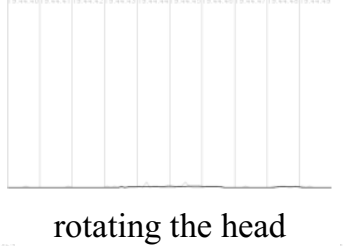

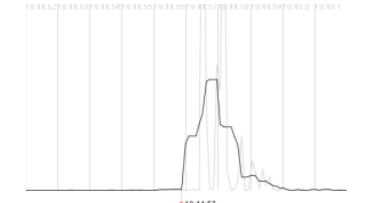

rotating the whole body left

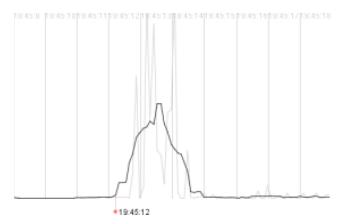

rotating the whole body right

Fig. 6. Examples for movements and their associated value $b_{t}$ over time 


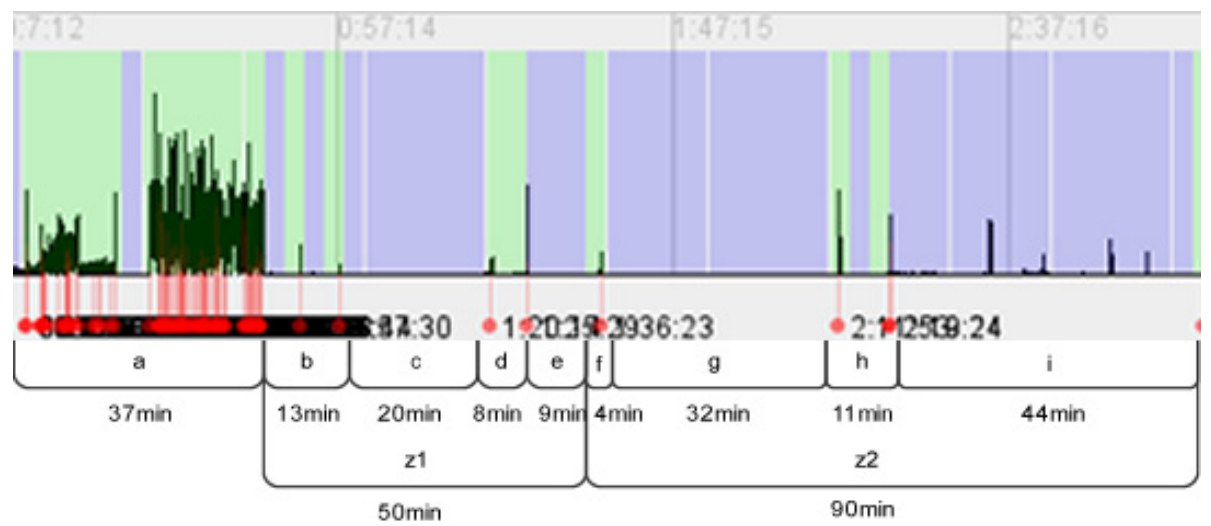

Fig. 7. Movement data over three hours in night one

- Strong response - lying down and standing up (1), rotating whole body (7), horizontal leg movement (4)

- Medium response - horizontal arm movement (2), lifting legs (5)

- Weak response - lifting arms (3), rotating head (6)

The second evaluation we performed was detecting movements in the sleep of a single person over three nights. Fig. 3 gives an example of three hours of the first night. We follow the methods presented by Salmi and Leinonen [13] to group the epochs and analyze the resulting data set. The exemplary analysis for the three hours shown in Fig. 3 results in the following sleep phases: (a) very active wake phase of $37 \mathrm{mi}$ nutes, (b) fallen asleep and spending 13 minutes in NREM phases 1 and 2, (c) about 20 minutes of deep sleep, (d) going back to phases 2 and 1, (e) first REM phase of 9 minutes, (f) approximately 4 minutes of light sleep, (g) deep sleep phase, (h) potential REM phase of 11 minutes, (i) potential light sleep and REM sleep.

The cycles $z 1$ and $z 2$ denote full sleep cycles with the person moving from deep sleep to REM and back again. The first has a duration of 50 minutes and the second of 90 minutes.
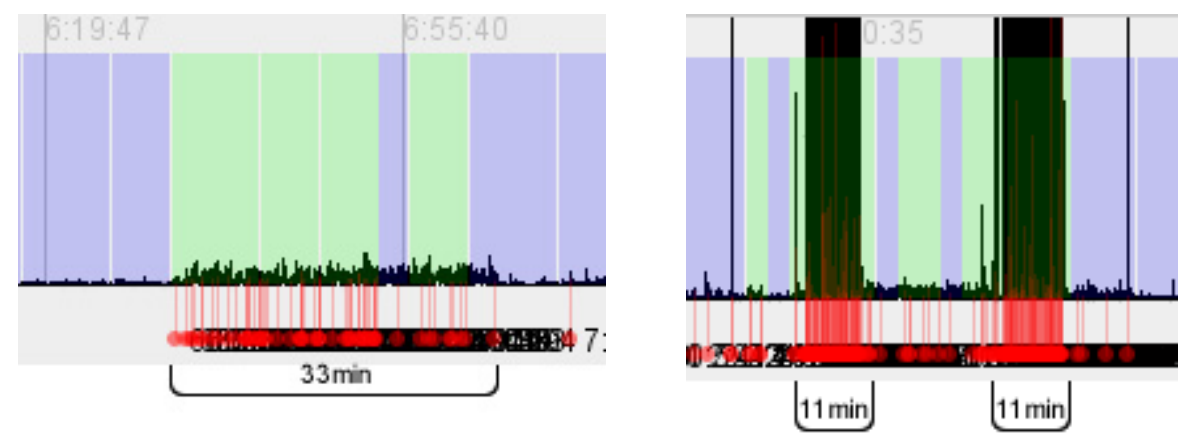

Fig. 8. Anomalies in the second (left) and third (right) night 
In the second and third night we were able to monitor some anomalies; i.e. atypical phases of very high activity as shown in Fig. 4. The anomaly of night 2 shows a period of constant activity over 33 minutes. The subject was most likely in a restless wake phase. The anomaly of night 3 shows to very intensive phases of sensor activity over 11 minutes each. The severity can't be attributed to regular movements. We assume that the sensors were either affected by some external influence, e.g. irregular power supply or there was an error in the data processing that could not be reproduced. While this temporarily disturbed the sleep phase detection, the system recovered later in the night.

Conclusively the system in most cases to reliably discern between wake, active sleep and quiet sleep allowing us to reproduce sleep phases with similar accuracy as Salmi and Leinonen [13].

\section{Conclusion and Future Work}

On the previous pages we have presented MoviBed, a sensor-equipped bed for personal sleep analysis. Based on a platform for capacitive proximity sensing MoviBed is able to detect movement on the bed and associate it to different phases of sleep. We have presented a method to calculate movement measures from raw capacitive sensor values in order to associate different periods of time to phases of wakefulness, active sleep and quiet sleep. We have tested this method in an extended prototype both for the capability to distinguish different movements, as well as for sleep phase recognition. We were able to reproduce the results of Salmi and Leinonen that introduced this method of movement-based sleep phase recognition, without requiring disturbing measurement devices typically used in full-scale sleep laboratories. Therefore MoviBed marks an important step in enabling regular customers to apply some minor sleep analysis in their own homes.

Nonetheless MoviBed is but an intermediate step in this direction. We plan to rebuild the prototype using the OpenCapSense capacitive proximity sensing toolkit, that provides higher precision compared to the CapToolKit used in the current iteration [15]. We expect that this allows us to more precisely distinguish different movements and in addition get an idea about respiratory movements that allows additional reasoning about the current sleep phase [16]. This combination of body and respiratory movement furthermore allows detecting additional sleep disturbances, such as sleep apnea. Based on this future iteration we are planning to perform more precise benchmarking in a sleep laboratory to get quantitative measurement about system capabilities and reliability. This evaluation will include more users over a longer period of time, possibly also users with known sleep disorders. MoviBed can be easily integrated into home control environments to provide additional services, such as energy saving by shutting of appliances as soon as the user is falling asleep, or activating certain appliances as soon as he wakes up again. Another idea is to increase the number of sensors to allow reliably distinguishing between two persons on a single bed. Finally we would like to develop a portable version of MoviBed that will allow quick installation and tuning of the system in arbitrary bed configurations. 
Acknowledgments. We would like to thank all volunteers that participated in our study and provided valuable feedback for future iterations. This work was partially funded by EC Grant Agreement No. 610840.

\section{References}

1. Pilcher, J.J., Huffcutt, A.J.: Effects of sleep deprivation on performance: A meta-analysis. Sleep J. Sleep Res. Sleep Med. 19, 318-326 (1996)

2. Moser, D., Anderer, P., Gruber, G., Parapatics, S.: Sleep classification according to AASM and Rechtschaffen \& Kales: effects on sleep scoring parameters. Sleep (2009)

3. Littner, M.M., Kushida, C.: Practice parameters for the role of actigraphy in the study of sleep and circadian rhythms: an update for 2002. Sleep (2003)

4. Jones, C., Campbell, S., Zone, S.: Familial advanced sleep-phase syndrome: A shortperiod circadian rhythm variant in humans. Nat. Med. (1999)

5. Zimmerman, T.G., Smith, J.R., Paradiso, J.A., Allport, D., Gershenfeld, N.: Applying electric field sensing to human-computer interfaces. In: Proceedings of the SIGCHI Conference on Human Factors in Computing Systems - CHI 1995, pp. 280-287. ACM Press, New York (1995)

6. Braun, A., Hamisu, P.: Using the human body field as a medium for natural interaction. In: Proceedings of the 2nd International Conference on PErvsive Technologies Related to Assistive Environments - PETRA 2009, pp. 1-7. ACM Press, New York (2009)

7. Große-Puppendahl, T.A., Marinc, A., Braun, A.: Classification of User Postures with Capacitive Proximity Sensors in AAL-Environments. In: Keyson, D.V., et al. (eds.) AmI 2011. LNCS, vol. 7040, pp. 314-323. Springer, Heidelberg (2011)

8. Braun, A., Heggen, H.: Context recognition using capacitive sensor arrays in beds. Technik für ein selbstbestimmtes Leben - 5. Deutscher AAL-Kongress. VDE VERLAG GmbH, Berlin (2012)

9. Krejcar, O., Jirka, J., Janckulik, D.: Use of mobile phones as intelligent sensors for sound input analysis and sleep state detection. Sensors (2011)

10. AppZoo GmbH, http : / thewakeapp. com/ (retrieved January 27, 2014)

11. Schulz, H.: Rethinking sleep analysis. J. Clin. Sleep Med. 4, 99-103 (2008)

12. Wilde-Frenz, J., Schulz, H.: Rate and distribution of body movements during sleep in humans. Percept. Mot. Skills (1983)

13. Salmi, T., Leinonen, L.: Automatic analysis of sleep records with static charge sensitive bed. Electroencephalogr. Clin. Neurophysiol. 64, 84-87 (1986)

14. Wimmer, R., Kranz, M., Boring, S., Schmidt, A.: A Capacitive Sensing Toolkit for Pervasive Activity Detection and Recognition. In: Fifth Annu. IEEE Int. Conf. Pervasive Comput. Commun. PerCom 2007, pp. 171-180 (2007)

15. Grosse-Puppendahl, T., Berghoefer, Y., Braun, A., Wimmer, R., Kuijper, A.: OpenCapSense: A Rapid Prototyping Toolkit for Pervasive Interaction Using Capacitive Sensing. In: IEEE Int. Conf. Pervasive Comput. Commun. (March 18-22, 2013)

16. Guerrero-Mora, G., Elvia, P.: Sleep-wake detection based on respiratory signal acquired through a Pressure Bed Sensor. Engineering in Medicine and Biology Society, EMBC (2012) 